\title{
Association Policy for XML Query Answering By Mining Tree
}

\author{
Shambhu Kumar Singh ${ }^{1}$, Sachinkumar S. Patil ${ }^{2}$, Abhinay C. Deshmukh ${ }^{3}$, Suraj C. Jadhav ${ }^{4}$, Nilesh Rajput ${ }^{5}$ \\ Assistant. Professor, Department of Information Technology, SSBT COET Bambhori, Jalgaon, India \\ Student, Department of Information Technology, SSBT COET Bambhori Jalgaon, India ${ }^{2,3,4,5}$
}

\begin{abstract}
As we consider any database, then to retrieve the information in the files is an important issue. Mostly the files are arranged in proper manner by using the trees and No SQL database. This approach of TBAS (Tree Based Association Rules) plays an important role for reducing the retrieval time of query. So that, if the user wants to retrieve the information about any particular subject then it can be provided by the relational database in very less time because the information is stored in XML files i.e. the information is stored by using tags. In this way the retrieval time is reduces. This Intentional Knowledge of mined information is beneficial, and this is an unique way to manipulate both the structure and the content of the XML document and, exact answers to the user queries whenever it required.
\end{abstract}

Keywords: TBAS (Tree Based Association Rules), Intentional Knowledge, XML (Extensible Markup Language).

\section{INTRODUCTION}

The every database focus on retrieving data in minimum interval of time and for that the different strategies are been used. The one of the new technique is to store the data into XML files that is nothing but (Extensible Markup Language).In this current era the XML is widely used in information technology field as well as in internet application programming.

There is huge growth in the XML as per as the current trade of IT. It has ability to extract data or knowledge from the database [1]. In this mining tree based association rules for $\mathrm{xml}$ query answering, the data will store in the XML file so that it easier to retrieve data from the database and hence the response time will be improved.

The Mining Tree Based Association Rules for XML Query Answering project mainly used for the large databases of the social networking sites where the manipulation of data and information is an very important issue. As given in figure the whole analogy is divided in four parts viz. User interface, XML File, Relational Database and No SQL databases.

In application module the whole information is stored that is nothing but the whole information which is in terms of images and textual data. On the other hand the XML file which is responsible for storing the information in semi structured form. The structured information is stored in the relational databases by using the SQL Query. And in the No SQL database the Unstructured data is stored which enhances the complexity in the database.

XML file gives emphasis on semi structured information where as the main GUI deals with collection of the multimedia data. It is complex to handle the unstructured data and so we are going to stored information in the relational database that is nothing but structural data.

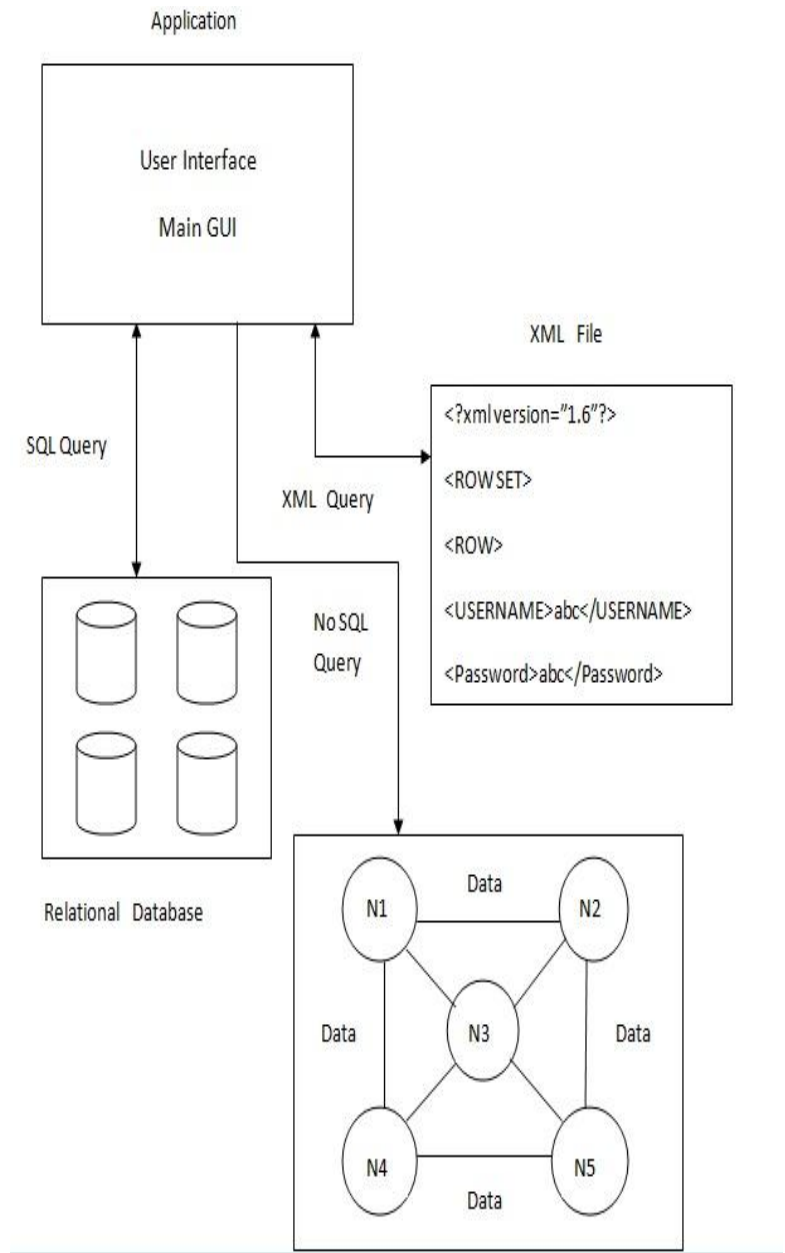

Fig 1. Block Diagram of Mining Tree Based Association Rules for XML Query Answering

\section{LITERATURE SURVEY}

Firstly, the concept of mining association rules implement in Fast Algorithm for Mining Association Rules in enormous Database, by R Agrawal and R Srikant, in 1994 
[1] for representing XML document in summarized manner. They propose various structured function which are apply in Apriori algorithm. In 2002 [2], T. Asai, K. Abe propose Apriori Algorithm which is responsible for creation of huge item set. This approach kindly executes the simple way for XML Query and answering. It is responsible for categorization of information present in semi structured and structured form. So that the drawback is removed by using XML files.

\section{FRAMEWORK}

The following figure shows the actual working of the XML Query Answering. In this system, the whole intentional information is going to be stored into the XML file by using the relational database, Here both the admin as well as user can be able to login into the application, Admin is responsible for the modification of the information and manipulate the whole database by using appropriate XML query where as user can only register himself by using unique user ID and password.

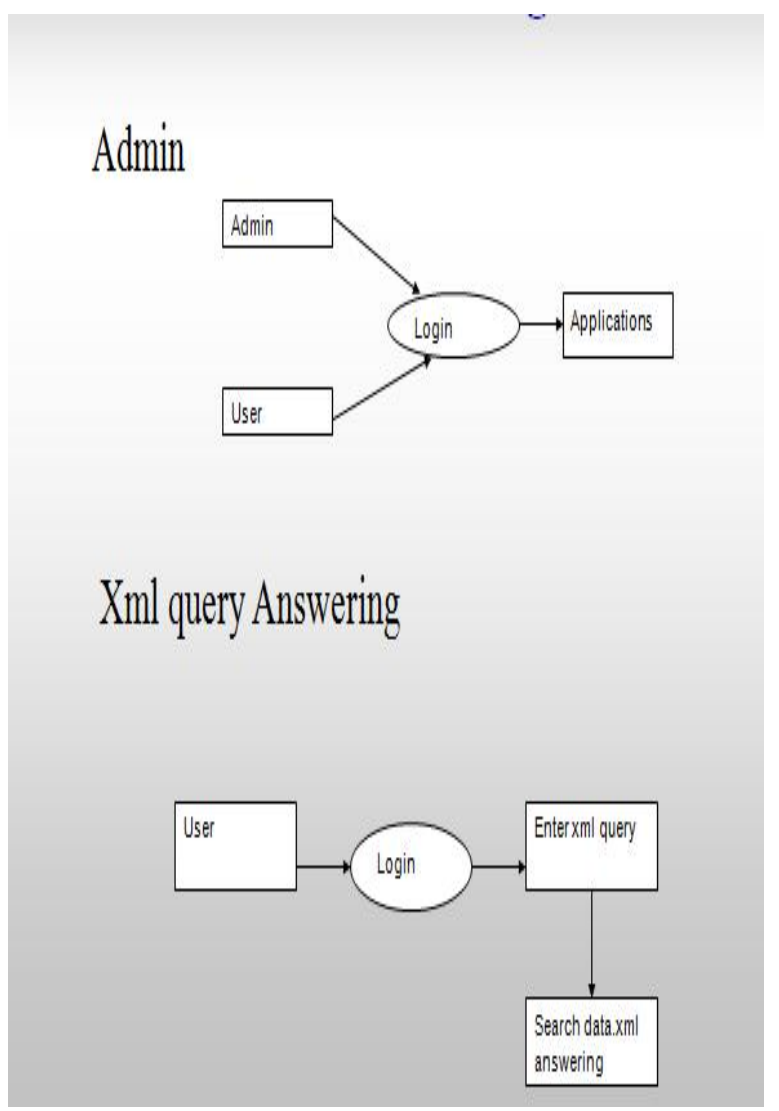

Fig 2. XML Query Answering System

On the basis of login system there are two ways for login. One is for admin and another one is for user. And by using login id the user can login into system and access the information which is stored in the XML files as shown in figure. The relational database is used for storing the intentional information which mostly gathers from the application. Every time the data is entered into the relational database and the updating is done .The whole unstructured information is stored into the proper way that is nothing but the XML document. The NO SQL databases Are also parts of the system which are used to store the unstructured data and which enhances the complexity of the database. The data is stored in the NO SQL database by using the NO SQL query. Among the each node the data is stored and nodes are connected to each other as shown in figure.

\section{CONCLUSION}

The current databases are very complex schemas. The manipulation of multimedia data is really challenging job. So to improve the response query time the data is stored in XML file and removes the complexity of database automatically[2]. The each and every module present in system is responsible for maintaining the information in the XML files. XML Files are directly stored the information so that the user get the right information on right time. The major advantage of the XML Query Answering is that user can retrieve the information in very less time. The goal of this project is to provide a way storing intentional information in the structured and semi structured form hence not only the complexity of database is removed but also the query retrievals time is reduced.

\section{REFERENCES}

[1] R. Agrawal and R. Srikant, "Fast Algorithms for Mining Association Rules in Large Databases," Proc. 20th Int'l Conf. Very Large Data Bases, pp. 478-499, 1994.

[2] T. Asai, K. Abe, S. Kawasoe, H. Arimura, H. Sakamoto, and S.Arikawa, "Efficient Substructure Discovery from Large Semi Structured Data,” Proc. SIAM Int'l Conf. Data Mining, 2002.

[3] D. Barbosa, L. Mignet, and P. Veltri, "Studying the XML Web: Gathering Statistics from an XML Sample,"WorldWideWeb, vol. 8 no. 4, pp. 413-438, 2005.

[4] Y. Chi, Y. Yang, Y. Xia, and R.R. Muntz, "CMTreeMiner: Mining both Closed and Maximal Frequent Subtrees," Proc. Eighth Pacific Asia Conf. Knowledge Discovery and Data Mining, pp. 63-73, 2004.

[5] C. Combi, B. Oliboni, and R. Rossato, "Querying XML Documents by Using Association Rules," Proc. 16th Int'1 Conf. Database and Expert Systems Applications, pp. 1020-1024, 2005

[6] Evfimievski, R. Srikant, R. Agrawal, and J. Gehrke, "Privacy Preserving Mining of Association Rules," Proc. Eighth ACM Int'l Conf. Knowledge Discovery and Data Mining, pp. 217-228, 2002.

[7] L. Feng, T.S. Dillon, H. Weigand, and E. Chang, "An XMLEnabled Association Rule Framework Applications pp,88_97,2003

[8] World Wide Web Consortium, XQuery An XML Query Language, http://www.w3C.org/TR/xquery, 2007.

[9] World Wide Web Consortium, Extensible Markup Language (XML) 1.0, http://www.w3C.org/TR/REC-xml/, 1998

[10] J.W.W. Wan and G. Dobbie, "Extracting Association Rules from XML Documents Using XQuery," Proc. Fifth ACM Int'l Workshop pp. 94-97, 2003. 